\title{
Extra generations and discrepancies of electroweak precision data
}

\author{
V.A. Novikov, L.B. Okun, \\ ITEP, Moscow, Russia \\ A.N. Rozanov, \\ CPPM, IN2P3, CNRS, Univ. Mediteranee, Marseilles, France \\ and ITEP, Moscow, Russia \\ M.I. Vysotsky \\ ITEP, Moscow, Russia
}

\begin{abstract}
It is shown that additional chiral generations are not excluded by the latest electroweak precision data if one assumes that there is no mixing with the known three generations. In the case of "heavy extra generations", when all four new particles are heavier than $Z$ boson, quality of the fit for the one new generation is as good as for zero new generations (Standard Model). In the case of neutral leptons with masses around 50 $\mathrm{GeV}$ ("partially heavy extra generations") the minimum of $\chi^{2}$ is between one and two extra generations.
\end{abstract}

Two years ago in paper [1] we analyzed bounds from the electroweak precision data on the non-decoupled New Physics in a form of additional heavy quark-lepton generations. It was shown that while the case of all four new fermions ( $U$ and $D$ quarks, neutral lepton $N$ and charged lepton $E$ ) heavier than $Z$ boson was excluded at $2.5 \sigma$ level, existence of new generations with relatively light neutral lepton $N\left(m_{N} \approx 50 \mathrm{GeV}\right)$ was allowed. At that time quality of Standard Model (SM) fit of the data was very good, $\chi^{2} / n_{\text {d.o.f. }}=15 / 14$. At the time of Osaka Conference, summer 2000, nothing radical happened but $\chi^{2}$ became $21 / 13$ and the level at which one extra heavy generation was excluded went down to $2 \sigma$ [2]. However the latest precision data announced summer 2001 [3] has changed the situation: the fit is still bad, 24/13, but now the presence of one heavy generation does not make the fit worse as compared with SM.

In Table 1 the LEPTOP fit of summer 2001 data is presented. There are two significant changes in comparison with previous data presented in Table 2:

1. Due to precision measurement of the cross-section of $e^{+} e^{-}$annihilation into hadrons in the interval 2-5 GeV at BES the error in $\bar{\alpha} \equiv \alpha\left(M_{Z}\right)$ is now two times smaller. (Following Electroweak Working Group (EWWG) we use result 四 though other estimates can be found in the literature as well);

2. Central value of $M_{W}$ is now bigger by a half of $\sigma$. 
Table 1: LEPTOP fit to electroweak observables. Year 2001. By italics we designate calculated (not measured) quantities.

\begin{tabular}{|l|l|r|r|r|}
\hline Source & Observable & Exp. data & LEPTOP fit & Pull \\
\hline & $\Gamma_{Z}[\mathrm{GeV}]$ & $2.4952(23)$ & $2.4966(16)$ & -0.6 \\
& $\sigma_{h}[\mathrm{nb}]$ & $41.540(37)$ & $41.480(14)$ & 1.6 \\
& $A_{F B}^{l}$ & $0.0171(10)$ & $0.0165(3)$ & 0.7 \\
& $R_{l}$ & $20.767(25)$ & $20.738(18)$ & 1.1 \\
& $A_{\tau}, A_{e}$ & $0.1465(33)$ & $0.1483(11)$ & -0.5 \\
& $R_{b}$ & $0.2165(7)$ & $0.2157(1)$ & 1.2 \\
& $R_{c}$ & $0.1719(31)$ & $0.1723(1)$ & -0.1 \\
& $A_{F B}^{b}$ & $0.0990(17)$ & $0.1040(8)$ & -2.9 \\
& $A_{F B}^{c}$ & $0.0685(34)$ & $0.0743(6)$ & -1.7 \\
& $s_{l}^{2}\left(Q_{F B}\right)$ & $0.2324(12)$ & $0.2314(1)$ & 0.9 \\
\hline & $A_{L R}$ & $0.1513(21)$ & $0.1483(11)$ & 1.4 \\
SLC I & $s_{l}^{2}\left(A_{L R}\right)$ & $0.2310(3)$ & $0.2314(1)$ & -1.4 \\
& $A_{b}$ & $0.9220(200)$ & $0.9349(1)$ & -0.6 \\
& $A_{c}$ & $0.6700(260)$ & $0.6684(5)$ & 0.1 \\
\hline LEP II, Tevatron & $m_{W}[\mathrm{GeV}]$ & $80.451(33)$ & $80.392(20)$ & 1.8 \\
& $s_{W}^{2}\left(m_{W}\right)$ & $0.2216(6)$ & & \\
\hline Tevatron & $s_{W}^{2}(\nu N)$ & $0.2255(21)$ & $0.2230(3)$ & 1.2 \\
& $m_{W}(\nu N)[\mathrm{GeV}]$ & $80.250(109)$ & & \\
& $m_{t}[\mathrm{GeV}]$ & $174.3(5.1)$ & $175.0(4.4)$ & -0.1 \\
\hline Fit & $m_{H}[\mathrm{GeV}]$ & & $79_{-29}^{+47}$ & \\
& $\hat{\alpha}_{s}$ & & $0.1182(27)$ & \\
\hline$e^{+} e^{-} \rightarrow$ hadrons & $\bar{\alpha}^{-1}$ & $128.936(49)$ & $128.918(45)$ & 0.4 \\
\hline & $\chi^{2} / n_{\text {d.o.f. }}$ & & $23.8 / 13$ & \\
\hline
\end{tabular}

The latter is the main cause for the relaxation of the bound on heavy extra generations.

Exclusion plot for the number $N_{g}$ of extra heavy generations is presented in Fig. 1.

To produce this plot we take $m_{D}=130 \mathrm{GeV}$ - the Tevatron lower bound on new quark mass; we use experimental 95\% C.L. bound on higgs mass $m_{H}>113$ $\mathrm{GeV}$ [3] and vary $\Delta m=\sqrt{m_{U}^{2}-m_{D}^{2}}$ and number of extra generations $N_{g}$. (In order to have two-dimensional plot we arbitrary assumed that $m_{N}=m_{U}$ and $m_{E}=m_{D}$; other choices do not change the obtained results drastically); $\chi^{2}$ minimum corresponds to unphysical point $N_{g}=0.5$. For $170 \mathrm{GeV}<m_{U}<200$ $\mathrm{GeV}$ we get the same quality of fit in the case $N_{g}=1$ as that for the SM $\left(N_{g}=0\right)$. In ref. [5] one can find a statement that extra heavy generations are excluded by the precision electroweak data. However, analysis performed in [5] refers to upper and lower parts of Fig. $1, \Delta m>200 \mathrm{GeV}$ and $\Delta m=0$, where the existence of new heavy generations is really strongly suppressed. This is not 
Table 2: LEPTOP fit to electroweak observables. Year 2000.

\begin{tabular}{|l|l|r|r|r|}
\hline Source & Observable & Exp. data & LEPTOP fit & Pull \\
\hline & $\Gamma_{Z}[\mathrm{GeV}]$ & $2.4952(23)$ & $2.4964(16)$ & -0.5 \\
& $\sigma_{h}[\mathrm{nb}]$ & $41.541(37)$ & $41.479(15)$ & 1.7 \\
& $A_{F B}^{l}$ & $0.0171(10)$ & $0.0164(3)$ & 0.7 \\
& $R_{l}$ & $20.767(25)$ & $20.739(18)$ & 1.1 \\
LEP I & $A_{\tau}, A_{e}$ & $0.1467(32)$ & $0.1480(13)$ & -0.4 \\
& $R_{b}$ & $0.2165(7)$ & $0.2157(1)$ & 1.2 \\
& $R_{c}$ & $0.1709(34)$ & $0.1723(1)$ & -0.4 \\
& $A_{F B}^{b}$ & $0.0990(20)$ & $0.1038(9)$ & -2.4 \\
& $A_{F B}^{c}$ & $0.0689(35)$ & $0.0742(7)$ & -1.5 \\
& $s_{l}^{2}\left(Q_{F B}\right)$ & $0.2321(10)$ & $0.2314(2)$ & 0.7 \\
\hline & $A_{L R}$ & $0.1514(22)$ & $0.1480(16)$ & 1.5 \\
SLC & $s_{l}^{2}\left(A_{L R}\right)$ & $0.2310(3)$ & $0.2314(2)$ & -1.5 \\
& $A_{b}$ & $0.9110(250)$ & $0.9349(1)$ & -1.0 \\
& $A_{c}$ & $0.6300(260)$ & $0.6683(6)$ & -1.5 \\
\hline LEP II, Tevatron & $m_{W}[\mathrm{GeV}]$ & $80.434(37)$ & $80.397(23)$ & 1.0 \\
& $s_{W}^{2}\left(m_{W}\right)$ & $0.2219(7)$ & & \\
\hline Tevatron & $s_{W}^{2}(\nu N)$ & $0.2255(21)$ & $0.2231(2)$ & 1.1 \\
& $m_{W}(\nu N)[\mathrm{GeV}]$ & $80.250(109)$ & & \\
& $m_{t}[\mathrm{GeV}]$ & $174.3(5.1)$ & $174.0(4.2)$ & 0.1 \\
\hline Fit & $m_{H}[\mathrm{GeV}]$ & & $55_{-26}^{+45}$ & \\
& $\hat{\alpha}_{s}$ & & $0.1183(27)$ & \\
\hline$e^{+} e^{-} \rightarrow$ hadrons & $\bar{\alpha}^{-1}$ & $128.878(90)$ & $128.850(90)$ & 0.3 \\
\hline & $\chi^{2} / n_{\text {d.o.f. }}$ & & $21.4 / 13$ & \\
\hline
\end{tabular}

the case for the central part of Fig. $1(\Delta m \approx 150 \mathrm{GeV})$.

Two heavy generations are excluded at more than $3 \sigma$ level. Nevertheless, two and even three "partially heavy" generations are allowed when neutral fermions are relatively light, $m_{N} \simeq 55 \mathrm{GeV}$ (see Fig. 2). Using all existing LEP II statistics on the reactions $e^{+} e^{-} \rightarrow \gamma+\nu \bar{\nu}, \gamma+N \bar{N}$ in dedicated search one can exclude 3 "partially heavy" generations which contain such a light $N$ at a level of $3 \sigma$ (see [6]), while one or even two such generations may exist.

The cause of disappearance of the suppression of extra heavy generations which existed in the early data is the contradiction in description of modern data on $M_{W}$ and $s_{l}^{2}$ in the framework of SM. The point is that the higgs mass, a free parameter of the SM, has the following values being extracted from these observables:

$$
\begin{aligned}
\left(m_{W}\right)_{\text {LEPII,Tevatron,NuTeV }} & =80.428(32) \mathrm{GeV} \Rightarrow \\
& \Rightarrow m_{H}=50_{-35}^{+50} \mathrm{GeV},
\end{aligned}
$$




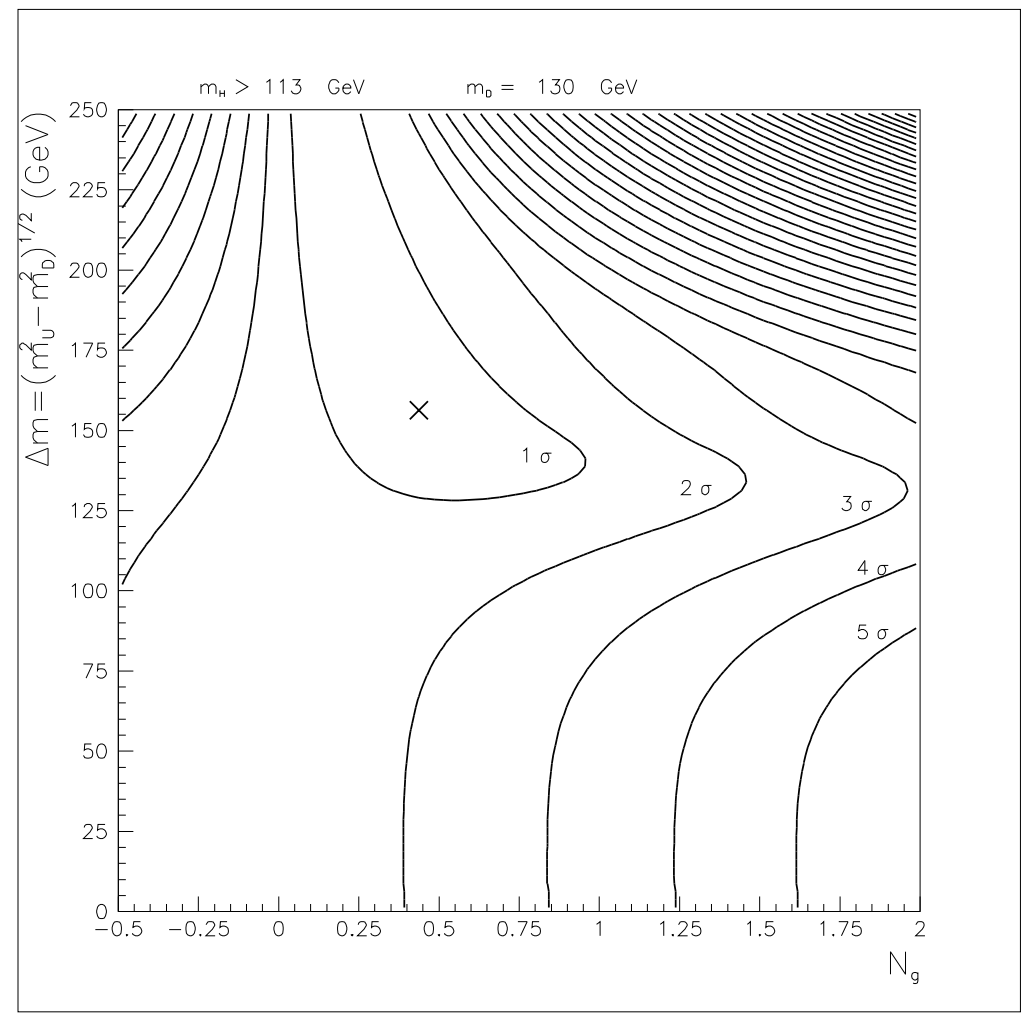

Figure 1: Exclusion plot for heavy extra generations with the input: $m_{D}=$ $m_{E}=130 \mathrm{GeV}, m_{U}=m_{N} . \chi^{2}$ minimum shown by cross corresponds to $\chi^{2} / n_{\text {d.o.f. }}=22.2 / 12, N_{g}=0.4, \Delta m=160 \mathrm{GeV}, m_{H}=116 \mathrm{GeV} . N_{g}$ is the number of extra generations. Borders of regions show domains allowed at the level $1 \sigma, 2 \sigma$, etc.

$$
\begin{aligned}
\left(s_{l}^{2}\right)_{\text {LEPI,SLAC }} & =0.23140(15) \Rightarrow \\
& \Rightarrow m_{H}=150_{-50}^{+75} \mathrm{GeV} .
\end{aligned}
$$

$N_{g}=0.5$ reduces the contradiction between the two values of $m_{H}$. Nevertheless the resulting $\chi^{2}$ does not improve drastically and this is due to another "defect" of precision data: the discrepancy between the average value of $s_{l}^{2}$ extracted from pure leptonic measurements and its value from events with hadrons in final state [3]:

$$
\begin{array}{cc} 
& s_{l}^{2} \\
\text { Leptons } & 0.23113(21) \\
\text { Hadrons } & 0.23230(29)
\end{array}
$$

These $3.3 \sigma$ difference is the root of poor quality of the SM fit. The value of hadronic contribution to $s_{l}^{2}$ in $(3)$ is dominated by very small uncertainty of 


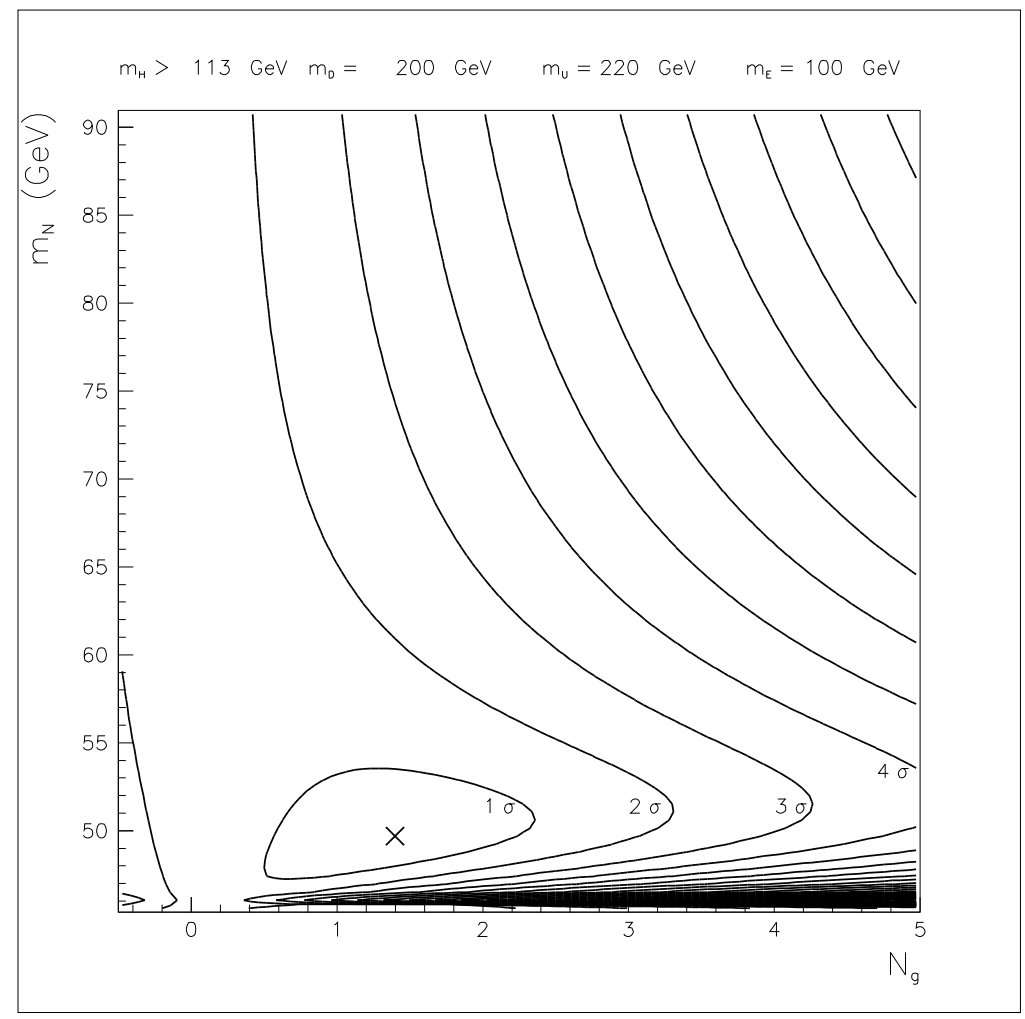

Figure 2: Exclusion plot for the number of partially heavy extra generations with light neutral lepton $N$. On horizontal axis the number of extra generations $N_{g}$, on vertical axis - the mass of the neutral lepton $m_{N}$. The input: $m_{U}=220$ $\mathrm{GeV}, m_{D}=200 \mathrm{GeV}, m_{E}=100 \mathrm{GeV}$. At the minimum $\chi^{2} / n_{\text {d.o.f. }}=21.6 / 12$, $N_{g}=1.4, m_{N}=50 \mathrm{GeV}, m_{H}=116 \mathrm{GeV}$. The spectacular behaviour of lines at the bottom of this figure as well as Fig. 4 is caused by the threshold singularity. This singularity must manifest itself also in the $Z$ lineshape. We have not studied it because according to experimental data by LEP collaborations on the emission of initial state bremsstrahlung photon $m_{N}>50 \mathrm{GeV}$ at $95 \%$ c.l. [6, (7] and the effect at such distance above threshold is not prominent. 
the forward-backward asymmetry in reaction $\quad e^{+} e^{-} \rightarrow Z \rightarrow b \bar{b}$. According to Table 1

$$
\left(A_{F B}^{b}\right)_{\exp }=0.0990(17) \text {. }
$$

One can question whether such a good accuracy can be obtained in the analysis of hadronic jets production. Another value of $A_{F B}^{b}$ can be obtained by multiplying measured at SLAC beauty asymmetry $A_{b}$ and leptonic asymmetry $A_{l}$. Then

$$
A_{F B}^{b}=\frac{3}{4} A_{b} A_{l}=0.1038(25) .
$$

The number (5) differs from (田), but nicely coincides with the SM fit: $0.1040(8)$ (see Table 1).

Let us assume following Chanowitz [8] that $A_{F B}^{b}$ has larger uncertainty than given in Eq. (4) and look to what consequences with respect to extra generations this hypothesis will lead. If we multiply experimental uncertainties of $A_{F B}^{b}$ and $A_{F B}^{c}$, which are strongly correlated, by a factor 10 , the quality of SM fit improves drastically: $\chi^{2} / n_{\text {d.o.f. }}$ shifts from $23.8 / 13$ to $10.9 / 13$ and simultaneously one heavy extra generation becomes excluded at the level of $2.5 \sigma$ (see Fig. 3).

However, a serious problem arises: it is just $A_{F B}^{b}$ given by Eq. (4) which pushes $m_{H}$ to larger values. With our modification of experimental results on $A_{F B}^{b}$ and $A_{F B}^{c}$ the SM fit gives:

$$
m_{H}=42_{-18}^{+30} \mathrm{GeV},
$$

well below modern LEP II bound: $m_{H}>113 \mathrm{GeV}$, a substantial trouble for the SM. In case the constraint $m_{H}>113 \mathrm{GeV}$ is imposed, we get: $m_{H}=116_{-2}^{+15}$ $\mathrm{GeV}, \chi^{2} / n_{\text {d.o.f. }}=14.5 / 14$. What concerns partially heavy extra generations, they nicely fit the data even with ten times enlarged uncertainties of $A_{F B}^{b}$ and $A_{F B}^{c}$, see Fig. 4. At both minima in this Figure $\chi^{2} / n_{\text {d.o.f. }} \simeq 13 / 12$, while $m_{H} \simeq 116 \mathrm{GeV}$ due to the imposed constraint $m_{H}>113 \mathrm{GeV}$. Without this constraint $m_{H}$ drops to $\sim 40 \mathrm{GeV}$, while $\chi^{2} / n_{\text {d.o.f. }} \simeq 10.1 / 11$ at $N_{g}=0.9$, $m_{N}=53 \mathrm{GeV}$. (The various values of $n_{\text {d.o.f. }}$. stems from unconstrained or constrained value of $m_{H}$ and to additional parameters $m_{N}$ and $N_{g}$ in case of New Physics.)

In the recent paper 10 it was noted that SUSY extension of Standard Model with light sneutrinos with masses in the range $55-80 \mathrm{GeV}$ is allowed by precision data and pushes higgs mass to larger values. $\left(A_{F B}^{b}\right.$ was neglected there as well). This might be a strong indication in favor of light SUSY particles.

\footnotetext{
${ }^{1}$ Another way to resolve situation with $A_{F B}^{b}$ is to assume that there exist New Physics contributions to $Z b \bar{b}$ couplings $g_{V}^{b}$ and $g_{A}^{b}$. Since in the expression for $A_{F B}^{b}$ the corrections to $g_{V}^{b}$ and $g_{A}^{b}$ are multiplied by small factor $g_{V}^{e}$ they should be large, so they must appear at the tree level. Also $Z \rightarrow b \bar{b}$ width proportional to $\left(g_{A}^{b}\right)^{2}+\left(g_{V}^{b}\right)^{2}$ should not noticeably change since $R_{b} \equiv \Gamma_{Z \rightarrow b \bar{b}} / \Gamma_{Z}$ is at present in good agreement with SM fit, see Table 1 . In recent paper 99 inclusion of additional bottom-like heavy quarks with vector currents is suggested to resolve the discrepancy (3).
} 


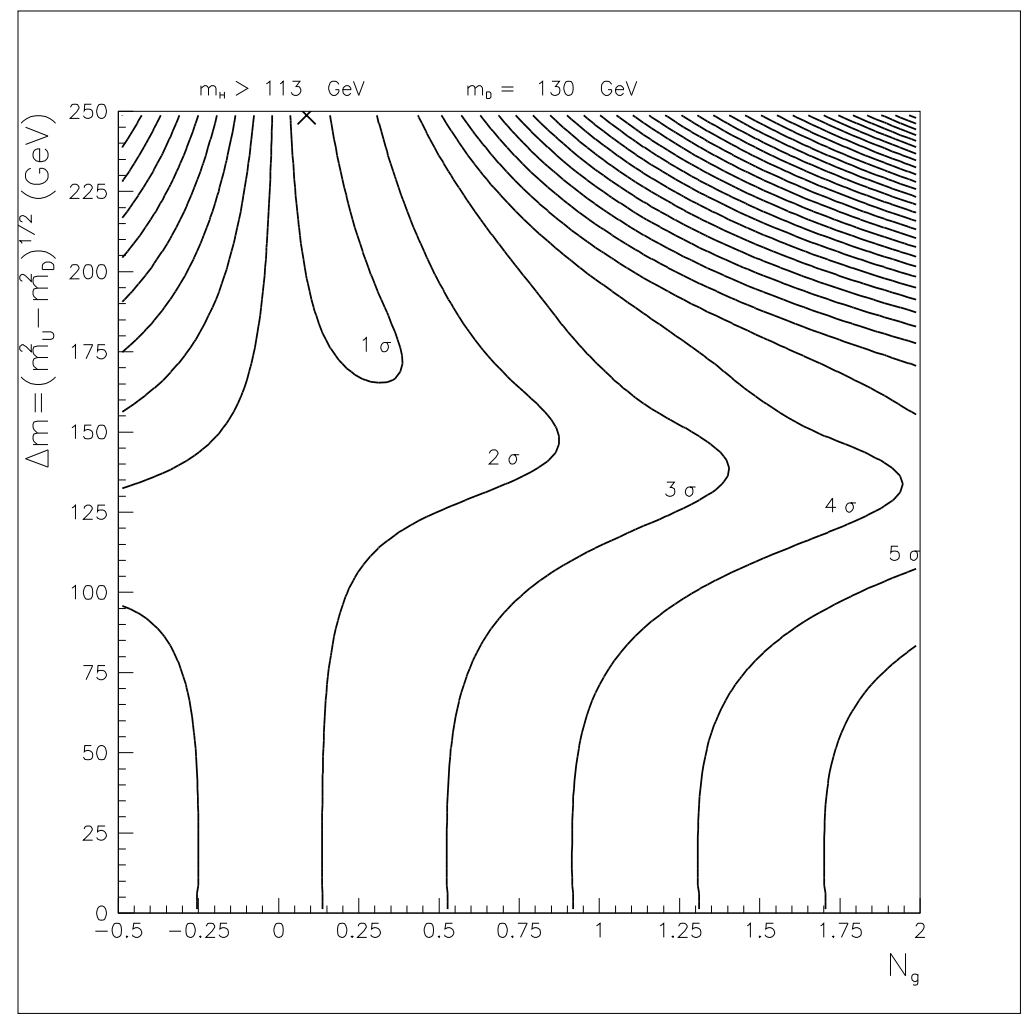

Figure 3: Exclusion plot for heavy extra generations with 10 times enlarged errors in $A_{F B}^{b}$ and $A_{F B}^{c}$ with the input $m_{D}=m_{E}=130 \mathrm{GeV}, m_{U}=m_{N}$. $\chi^{2}$ minimum is at the upper border of the Fig., where $\chi^{2} / n_{\text {d.o.f. }}=11.8 / 12$, $N_{g}=0.1, \Delta m=248 \mathrm{GeV}, m_{H}=116 \mathrm{GeV}$. 


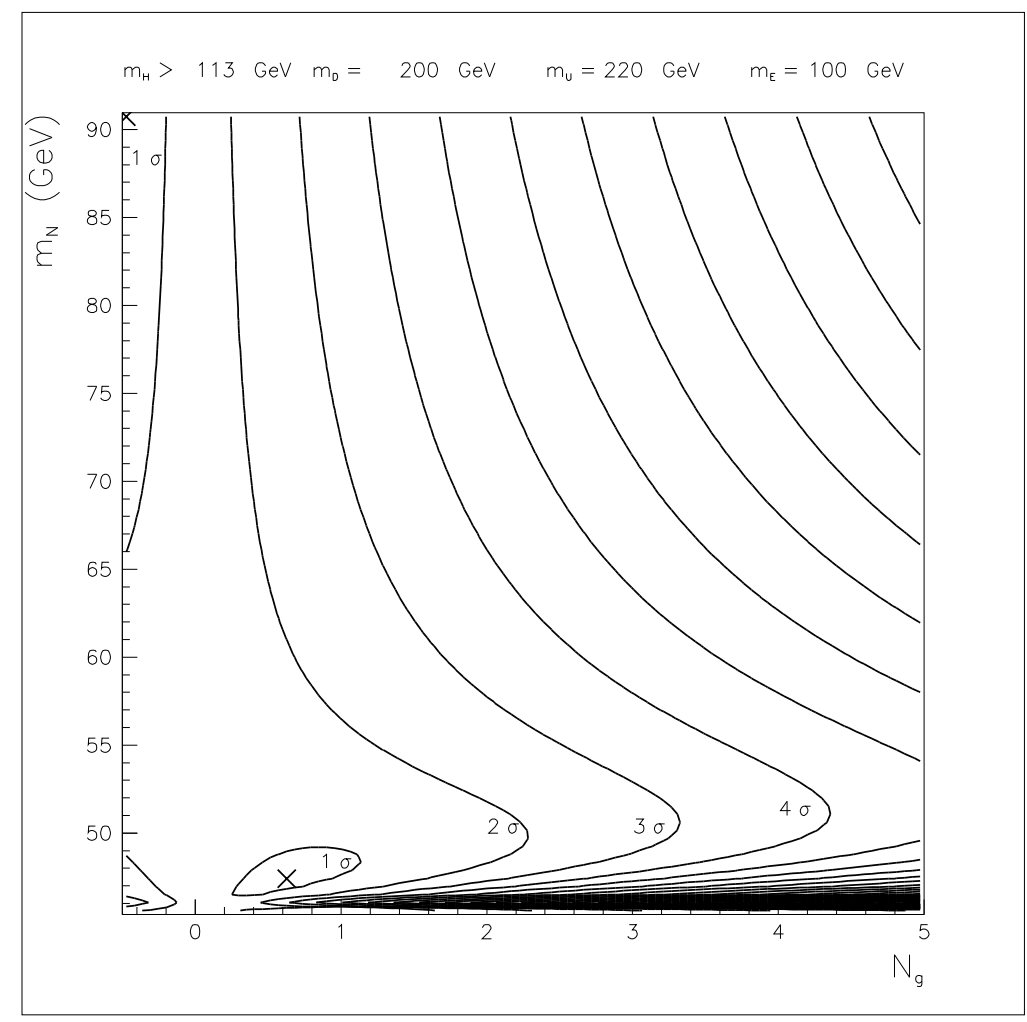

Figure 4: Exclusion plot for partially heavy extra generations with 10 times enlarged errors in $A_{F B}^{b}$ and $A_{F B}^{c}$ with the input $m_{D}=200 \mathrm{GeV}, m_{U}=220$ $\mathrm{GeV}, m_{E}=100 \mathrm{GeV}$. Two local $\chi^{2}$ minima are shown. At the first minimum $\chi^{2} / n_{\text {d.o.f. }}=12.4 / 12, N_{g}=-0.5, m_{N}=90 \mathrm{GeV}, m_{H}=116 \mathrm{GeV}$ (see upper left corner of the plot). At the second minimum $\chi^{2} / n_{\text {d.o.f. }}=13.1 / 12, N_{g}=0.6$, $m_{N}=48 \mathrm{GeV}, m_{H}=116 \mathrm{GeV}$.

The presence of new particles is important for production and decay of higgs. New heavy quarks considerably enhance higgs production at Tevatron and LHC through gluon fusion which should help to discover this particle 11]. If the decay of higgs into a pair of neutral leptons is kinematically allowed it will dominate, so that a moderately heavy higgs will decay invisibly [12]. At LEP II the invisibly decaying higgs is excluded almost at the same level as the SM higgs by missing mass method [13]. Contrary to that the LHC will look for visible decay modes of higgs. If the branching ratios of the latter are small the search will be not easy.

We are grateful to A. Olshevsky for providing ref. [3] and to P.S. Bambade and M. Stanitzki for providing ref. [13].

L.O., A.R. and M.V. are grateful to CERN EP and TH for hospitality; L.O., V.N. and M.V. were partly supported by RFBR grant No. 00-15-96562. 
P.S. After this paper had been completed a new result for $s_{W}^{2}(\nu N)$ and hence for $m_{W}(\nu N)$ was published by $\mathrm{NuTeV}$ collaboration [14]:

$$
\begin{gathered}
s_{W}^{2}(\nu N)=0.2277(17), \\
m_{W}(\nu N)=80.140(80) .
\end{gathered}
$$

The new value of $m_{W}(\nu N)$ differs from $m_{W}$ measured by LEP II and Tevatron by $3.7 \sigma$ and leads to a pull of 2.8 instead of 1.2 (see Table 1) aggravating the discrepancy. Using the same procedure as for Table 1 we get:

$$
\begin{gathered}
m_{H}=86_{-32}^{+51} \mathrm{GeV}, \\
\chi^{2} / n_{\text {d.o.f. }}=30.3 / 13 .
\end{gathered}
$$

The influence of the new $\mathrm{NuTeV}$ data on the limits on extra generations, as well as the change of LEPTOP code accounting for the new NuTeV procedure of extracting $s_{W}^{2}(\nu N)$ will be discussed elsewhere.

We are grateful to V.Rubakov for providing ref. 14.

\section{P.P.S.}

As a response to the appearance of this article on hep/ph H.-J.He kindly brought to our attention ref. [15], in which the problem of extra generations has been considered in a framework of the models with two and one Higgs doublets. In latter case the results of ref. [15] could be compared with ours. According to ref.[15], the $500 \mathrm{GeV}$ higgs, if accompanied by fourth generation, does not contradict the electroweak precision data. In order to check this statement we made special LEPTOP runs assuming $m_{H}=500 \mathrm{GeV}$ and $N_{g}=1$. We found that for certain fixed values of quark and lepton masses the $\chi^{2}$ of the fits with heavy higgs is even better than in the SM. For example, for $m_{N}=55$ $\mathrm{GeV}, m_{E}=200 \mathrm{GeV}, m_{U}=130 \mathrm{GeV}, m_{D}=130 \mathrm{GeV}$, and $m_{H}=500 \mathrm{GeV}$ $\chi^{2} / n_{\text {d.o.f. }}=20.3 / 14$ which should be compared with $\chi^{2} / n_{\text {d.o.f. }}=23.8 / 13$ from Table 1. Let us note that we do not use S, T, U parametrization of oblique corrections which is well suited for heavy fermions but not for light ones (with masses of the order of $M_{Z}$ ). 


\section{References}

[1] M. Maltoni, V.A. Novikov, L.B. Okun, A.N. Rozanov, and M.I. Vysotsky, Phys. Lett. B476 (2000) 107.

[2] V.A. Ilyin, M. Maltoni, V.A. Novikov, L.B. Okun, A.N. Rozanov, and M.I. Vysotsky, in: Proceedings of the XXX International Conference on High Energy Physics, Osaka-2000, World Scientific, v.2, p. 1209-1212.

[3] M.W. Grünewald, Talk at LEP Physics Jamboree, CERN, July 10th, 2001; http://www.cern.ch/LEPEWWG.

[4] H. Burkhardt and B. Pietrzyk, LAPP-EXP-2001-03.

[5] J. Erler and P. Langacker, in: Review of Particle Physics, The European Physical Journal 15 (2000) 95.

[6] V.A. Ilyin et al., Phys. Lett. B503 (2001) 126.

[7] The ALEPH Collaboration, ALEPH 2001-010; CONF 2001-007 (2001);

P.Abreu et al, DELPHI Collaboration, Eur.Phys.J. C 16 (2000) 53;

M.Acciari et al., L3 Collaboration, Phys.Lett. B470 (1999) 268;

G.Abbiendi et al., OPAL Collaboration, Eur.Phys.J. C 14 (2000) 73;

see also the contributions to the Summer 2001 Conferences.

[8] M.S. Chanowitz, hep-ph/0104024 (2001).

[9] D. Choudhury, T.M.P. Tait and C.E.M. Wagner, hep-ph/0109097 (2001).

[10] G. Altarelli, F. Caravaglios, G.F. Giudice, P. Gambino, G. Ridolfi, JHEP 0106 (2001) 018.

[11] V.D. Barger and R.J.N. Philips, Collider Physics, Addison-Wesley (1997); E. Arik et al., CERN-ATLAS Internal Note, ATL-PHUS-98-125 (1998);

I.F. Ginzburg, I.P. Ivanov and A. Schiller, Phys. Rev. D60 (1999) 095001; E. Arik et al., hep-ph/0109037 (2001).

[12] D. Fargion, Yu.A. Golubkov, M.Yu. Khlopov, R.V. Konoplich, R. Migrani, JETP Lett. 69 (1999) 434; Pis'ma v ZhETP 69 (1999) 402;

V.A. Khoze, hep-ph/0105069.

[13] ALEPH, DELPHI, L3 and OPAL Collaborations, The LEP working group for Higgs boson searches; ALEPH 2001-36, DELPHI 2001-117, L3 note 2702, Opal Technical Note TN694; paper submitted to EPS01 in Budapest.

[14] NuTeV Collaboration, hep-ex/0110059.

[15] H.-J.He, N.Polonsky, and S.Su,hep-ph/01020144 v2 16 May 2001. 